\title{
PRACTICAL IMPLEMENTATION OF GENETICALLY DESIGNED COMPUTED-TORQUE/FUZZY-LOGIC CONTROLLERS FOR ROBOTIC MANIPULATORS
}

\author{
B Porter, \\ Department of Industrial and Manufacturing \\ Systems Engineering, \\ The University of Hong Kong, \\ Hong Kong. \\ e-mail: bporter@hkucc.hku.hk
}

\author{
N N Zadeh, \\ Research Institute for Design, Manufacture, and \\ Marketing, \\ University of Salford, \\ Salford M5 4WT, \\ England. \\ e-mail: n.n.zadeh@aeromech.salford.ac.uk
}

\begin{abstract}
In this paper, experimental results are presented which were obtained by testing a practical direct-drive two-link robotic manipulator. In these tests, the trajectory-tracking performance of a genetically optimized non-fuzzy computed-torque/proportional-plus-derivative controller is compared with that of two kinds of genetically designed computed-torque/fuzzy-logic controller. The experimental results are very encouraging and indicate that genetically designed computed-torque/fuzzy-logic controllers may be suitable for routine use with industrial robots.
\end{abstract}

\section{Introduction}

It was shown by Porter [1] that genetic algorithms (Holland [2], Goldberg [3]) provide a very effective means of solving various complex problems in control engineering. In the particular field of robotics, this general approach to the genetic design of control systems can be used to improve the robustness characteristics of computed-torque controllers (Craig [4]) for robotic manipulators. Thus, for example, it was shown by Porter and Zadeh [5] that genetic algorithms can be readily used to select the optimal proportional and derivative gain matrices in non-fuzzy computed-torque/proportional-plusderivative controllers for robotic manipulators. In addition, in order to improve the trajectory- tracking performance of such controllers in the presence of unmodelled dynamics (arising, for example, from unforseen changes in payload), Porter and Zadeh [5] introduced a new class of computed-torque/fuzzy-logic controller.
Indeed, it was shown by Porter and Zadeh [5] that genetic algorithms can be readily used to determine the optimal domains of the fuzzy sets embodied in the fuzzylogic components of such computed-torque/fuzzy-logic controllers. Furthermore, it was demonstrated by Porter and Zadeh [5] in simulation studies that these computedtorque/fuzzy-logic controllers are more robust than genetically designed non-fuzzy computed torque/proportional-plus-derivative controllers. Moreover, in more recent investigations, Porter and Zadeh [6] showed in simulation studies that even more robust computed-torque/fuzzy-logic controllers can be designed by using genetic algorithms to determine the optimal sets of fuzzy rules as well as the optimal domains of the associated fuzzy sets embodied in such controllers. These results of Porter and Zadeh [5] [6] thus demonstrate that genetic algorithms provide a very effective means of introducing fuzzy logic into the control algorithms embodied in computed-torque controllers, whilst retaining the well-proven computed-torque structure of such controllers.

However, in order to investigate the possible use of such genetically designed computed-torque/fuzzy-logic controllers with practical industrial robots, it is necessary to go beyond the simulation studies of Porter and Zadeh [5] [6]. In this paper, experimental results are therefore presented which were obtained by testing a practical direct-drive two-link robotic manipulator. In these tests, the trajectory-tracking performance of hardware/software implementations of each of the following three controllers was compared:

(i) $\mathrm{C}_{1}$, a non-fuzzy computed torque/proportionalplus-derivative controller for which the gain matrices are determined genetically; 
(ii) $\mathrm{C}_{2}$, a computed-torque/fuzzy-logic controller for which only the domains of the fuzzy sets are determined genetically;

(iii) $\mathrm{C}_{3}$, a computed-torque/fuzzy-logic controller for which the fuzzy rules as well as the domains of the fuzzy sets are determined genetically.

In these practical tests, it was found that the controller, $\mathrm{C}_{3}$, is better than the controller, $\mathrm{C}_{2}$, and that the controller, $\mathrm{C}_{2}$, is better than the controller, $\mathrm{C}_{1}$. These tests are very encouraging and indicate that genetically designed computed-torque/fuzzy-logic controllers may be sufficiently robust for routine use with industrial robots.

\section{Genetic Design Procedure}

\subsection{Computed-Torque/Proportional-Plus- Derivative Controllers}

These controllers are governed by control-law equation of the form

$$
\tau=\mathrm{M}(\theta)\left(\ddot{\theta}_{\mathrm{d}}+\mathrm{u}\right)+\mathrm{h}(\theta, \dot{\theta})
$$

where

$$
\mathrm{u}=\mathrm{K}_{1} \mathrm{e}+\mathrm{K}_{2} \dot{\mathrm{e}}
$$

In equations (1) and (2), $\tau \in \mathfrak{R}^{\mathrm{n}}$ is the vector of actuator torques, $\theta \in \mathfrak{R}^{\mathrm{n}}$ is the vector of joint angles, $\theta_{d} \in \mathfrak{R}^{\mathrm{n}}$ is the vector of desired joint angles, $e=\theta_{d}-\theta \in \mathfrak{R}^{n}$ is the vector of joint-angle errors, $M(\theta) \in \mathfrak{R}^{\mathrm{nxn}}$ is the inertia matrix, $h(\theta, \dot{\theta}) \in \Re^{\mathrm{n}}$ is the vector of centrifugal, coriolis, gravitational, and frictional torques, $\mathrm{u} \in \mathfrak{R}^{\mathrm{n}}$ is the output vector from the proportional-plus-derivative controller, $\mathrm{K}_{1} \in \mathfrak{R}^{\mathrm{nxn}}$ is the proportional gain matrix, and $\mathrm{K}_{2} \in \mathfrak{R}^{\mathrm{nxn}}$ is the derivative gain matrix.

The design problem is to determine the controller gain matrices, $\mathrm{K}_{1} \in \mathfrak{R}^{\mathrm{nxn}}$ and $\mathrm{K}_{2} \in \mathfrak{R}^{\mathrm{nxn}}$, so as to minimize the cost function

$$
\Gamma=\int_{0}^{\mathrm{T}}\|\mathrm{e}(\mathrm{t})\| \mathrm{dt}
$$

when the robotic manipulator performs a specified trajectory-tracking task of duration $\mathrm{T}$. It was shown by Porter and Zadeh [5] that this problem can be solved using genetic algorithms by representing each proportional-plus-derivative controller governed by equation (2) as a string of binary digits. In this way, the standard techniques of evolutionary computing readily yield the optimal gain matrices for the controller $C_{1}$.

\subsection{Computed-Torque/Fuzzy-logic Controllers}

These controllers are governed by control-law equations of the form (1) where

$$
u_{i}=k_{i}\left(e_{i}, \dot{e}_{i}\right) \quad(i=1,2, \ldots, n)
$$

are the decoupled outputs from $n$ fuzzy-logic controllers. Each of these $n$ fuzzy-logic controllers is governed by rules of the following form:

$$
\begin{aligned}
& \text { If } e_{i} \text { is } P_{i}{ }^{(j)} \text { and } \dot{e}_{i} \text { is } Q_{i}^{(k)} \text {, } \\
& \text { then } u_{i} \text { is } R_{i}^{(1)}(i=1,2, \ldots, n) \text {. }
\end{aligned}
$$

The entire sets of fuzzy sets in the $e_{i}, \dot{e}_{i}$, and $u_{i}$ spaces are, respectively,

$$
\begin{aligned}
& P_{i}=\left\{P_{i}^{(1)}, P_{i}^{(2)}, \ldots, P_{i}^{(p)}\right\}(i=1,2, . ., n), \\
& Q_{i}=\left\{Q_{i}^{(1)}, Q_{i}^{(2)}, \ldots, Q_{i}^{(q)}\right\}(i=1,2, . ., n),
\end{aligned}
$$

and

$$
R_{i}=\left\{R_{i}{ }^{(1)}, R_{i}^{(2)}, \ldots, R_{i}^{(r)}\right\}(i=1,2, \ldots, n)
$$

These entire sets of fuzzy sets are symmetric and are, respectively, defined on the domains $\left[-\alpha_{i},+\alpha_{i}\right]$, $\left[-\beta_{i},+\beta_{i}\right]$, and $\left[-\gamma_{i},+\gamma_{i}\right](i=1,2, \ldots, n)$. The design problem is to determine the set of fuzzy rules and the domains of the associated fuzzy sets so as to minimize the cost function (3) when the robotic manipulator performs a specified trajectory-tracking task of duration T.

It was shown by Porter and Zadeh [5] [6] that this problem can be solved using genetic algorithms by representing each fuzzy-logic controller governed by equation (4) as a string of binary digits. In this way, the standard techniques of evolutionary computing readily yield the optimal domains of the fuzzy sets for the controller $\mathrm{C}_{2}$, and the optimal set of fuzzy rules as well as the optimal domains of the fuzzy sets for the controller $C_{3}$. 


\section{Practical Test Results}

In order to evaluate the practical performance of the these controllers $\mathrm{C}_{1}, \mathrm{C}_{2}$, and $\mathrm{C}_{3}$, a series of tests was performed on a direct-drive two-link robotic manipulator located in the laboratories of the University of Salford. In each case, the controller was designed in the absence of payload; but each controller was required to perform its trajectory-tracking task both without payload and with payload.

In the case of the controller $\mathrm{C}_{1}$ (i.e., the non-fuzzy computed-torque/proportional-plus-derivative controller with genetically determined gains), the time-domain behaviour of the end effector in the $x-y$ plane when performing the task without payload is as shown in Fig. 1 (a); the associated value of the cost function is

$$
\Gamma_{1}=349 \times 10^{-4}
$$

owever, if the controller $C_{1}$ is required to perform the task with a payload of $4 \mathrm{~kg}$, the time-domain behaviour of the end effector in the $x-y$ plane is as shown in Fig. 1(b); the associated value of the cost function is

$$
\Gamma_{2}=449 \times 10^{-4}
$$

Similarly, in the case of the controller $\mathrm{C}_{2}$ (i.e., the computed-torque/fuzzy-logic controller with genetically determined fuzzy-set domains), the time-domain behaviour of the end effector in the $x-y$ plane when performing the trajectory tracking task without payload is as shown in Fig. 2(a); the associated value of the cost function is

$$
\Gamma_{3}=64 \times 10^{-4}
$$

However, if the controller $C_{2}$ is required to perform the task with a payload of $4 \mathrm{~kg}$, the time-domain behaviour of the end effector in the $x-y$ plane of is as shown in Fig. 2 (b); the associated value of the cost function is

$$
\Gamma_{4}=94 \times 10^{-4}
$$

Finally, in the case of the controller $\mathrm{C}_{3}$ (i.e., the computed-torque/fuzzy-logic controller with genetically determined fuzzy rules and fuzzy-set domains), the timedomain behaviour of the end effector in the $x-y$ plane when performing the trajectory tracking task without payload is as shown in Fig. 3(a); the associated value of the cost function is

$$
\Gamma_{5}=52 \times 10^{-4}
$$

However, if the controller $C_{3}$ is required to perform the task with a payload of $4 \mathrm{~kg}$, the time-domain behaviour of the end effector in the $x-y$ plane is as shown in Fig. 3(b); the associated value of the cost function is

$$
\Gamma_{6}=92 \times 10^{-4}
$$

It is evident from these test results that, for this typical trajectory-tracking task,

$$
\Gamma_{5}<\Gamma_{3}<\Gamma_{1}
$$

and

$$
\Gamma_{6}<\Gamma_{4}<\Gamma_{2}
$$

Therefore, for this task, the controller, $\mathrm{C}_{3}$, is better than the controller, $\mathrm{C}_{2}$, and the controller, $\mathrm{C}_{2}$, is better than the controller, $\mathrm{C}_{1}$.

\section{Conclusion}

In this paper, experimental results have been presented which were obtained by testing a practical direct-drive robotic manipulator. In these tests, the trajectory-tracking performance of a genetically optimised non-fuzzy computed-torque/proportional-plus-derivative controller has been compared with that of two kinds of genetically designed computed-torque/fuzzy-logic controller. These test results are very encouraging and indicate that genetically designed computed-torque/fuzzy-logic controllers may be sufficiently robust for routine use with industrial robots.

\section{References}

[1] B. Porter. Genetic design of control systems. J. SICE, vol 34, pp 393-402, 1995.

[2] J.H. Holland. Adaptation in Natural and Artificial Systems. University of Michigan Press, 1975.

[3] D.E. Goldberg. Genetic Algorithms in Search, Optimization and Machine Learning. Addison-Wesley, 1989.

[4] J. J. Craig. Introduction to Robotics. Addison-Wesley, 1989.

[5] B. Porter and N.N. Zadeh. Genetic design of computedtorque/fuzzy-logic controllers for robotic manipulators. Proc IEEE International Symposium on Intelligent Control, Monterey, California, USA, August 1995.

[6] B. Porter and N.N. Zadeh. Genetic rule induction in the design of computed-torque/fuzzy-logic controllers for robotic manipulators. Proc IEEE International Symposium on Intelligent Control, Dearborn, Michigan, USA, September 1996 . 


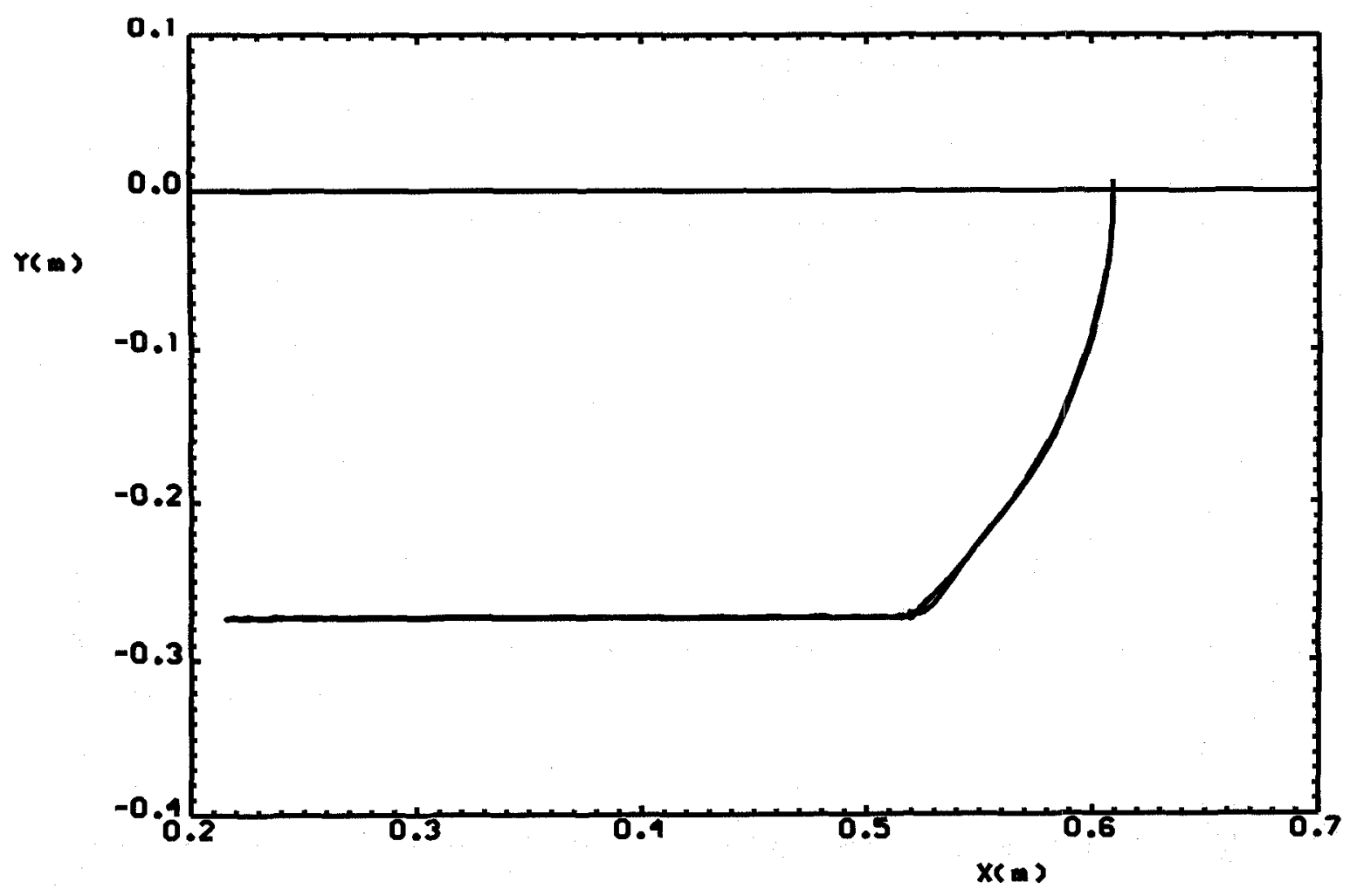

(a)

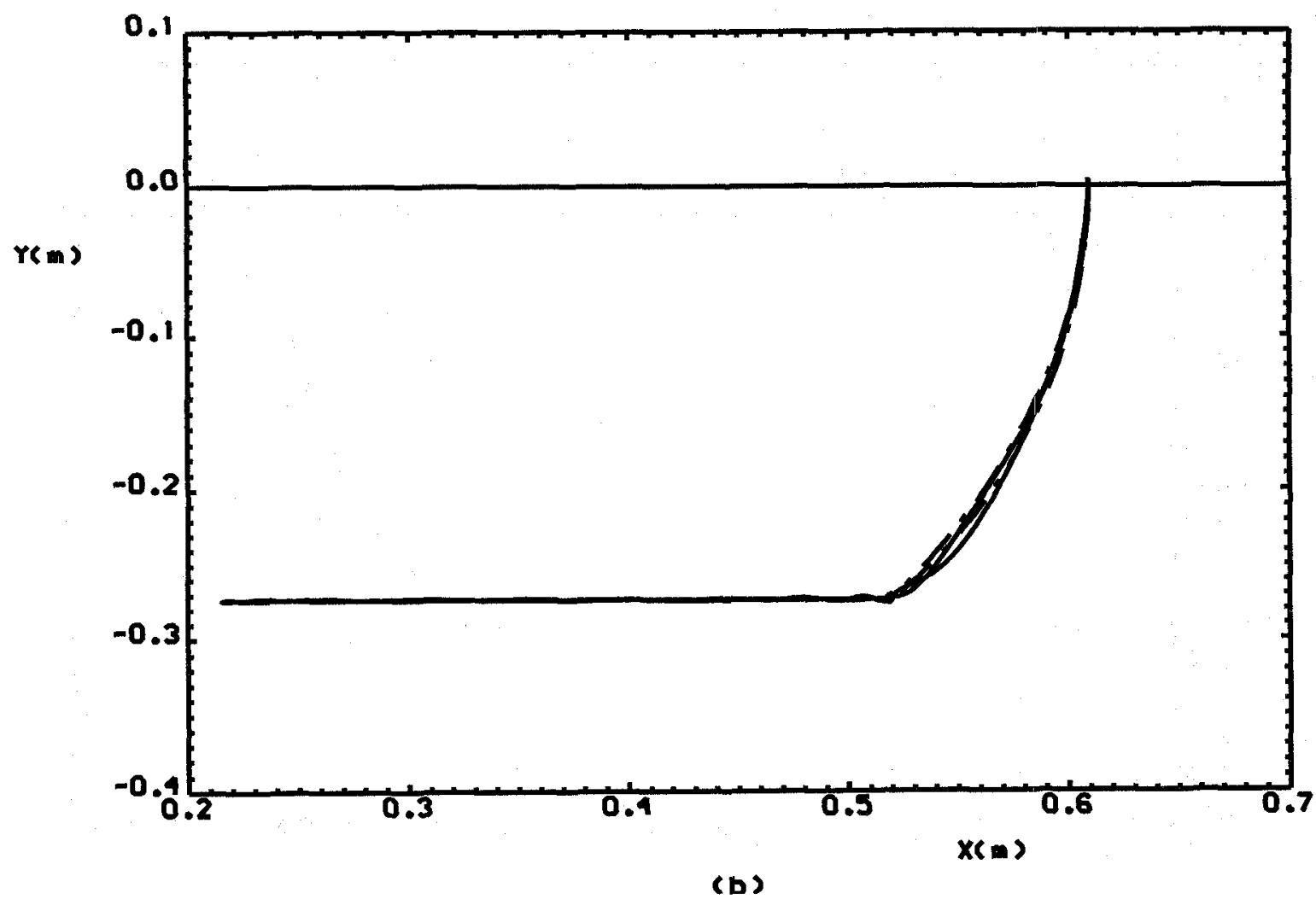

Figure 1: Work-space trajectory tracking performance (controller $\mathbf{C}_{1}$ )

(a) without payload (b) with payload 


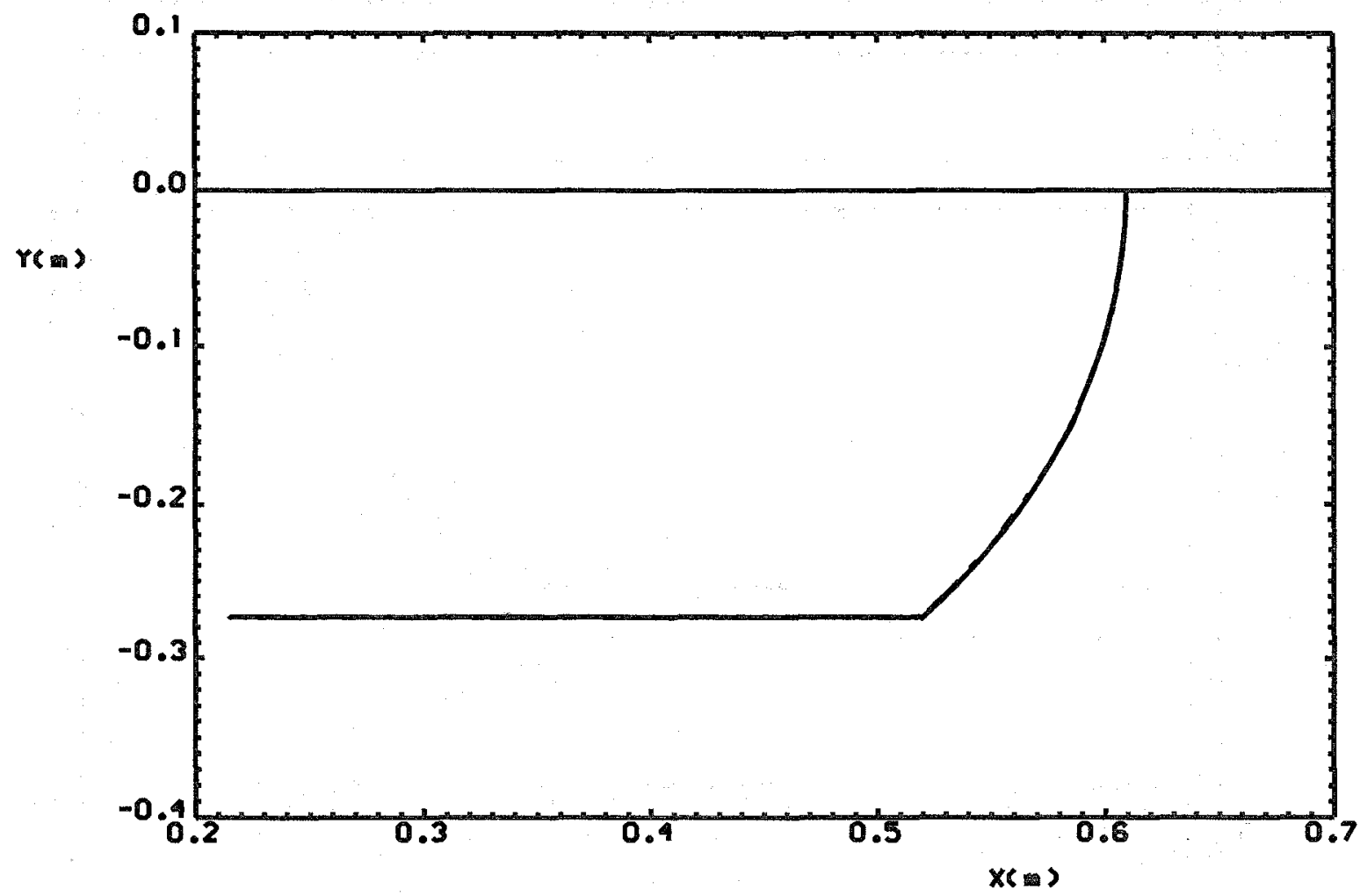

(a)

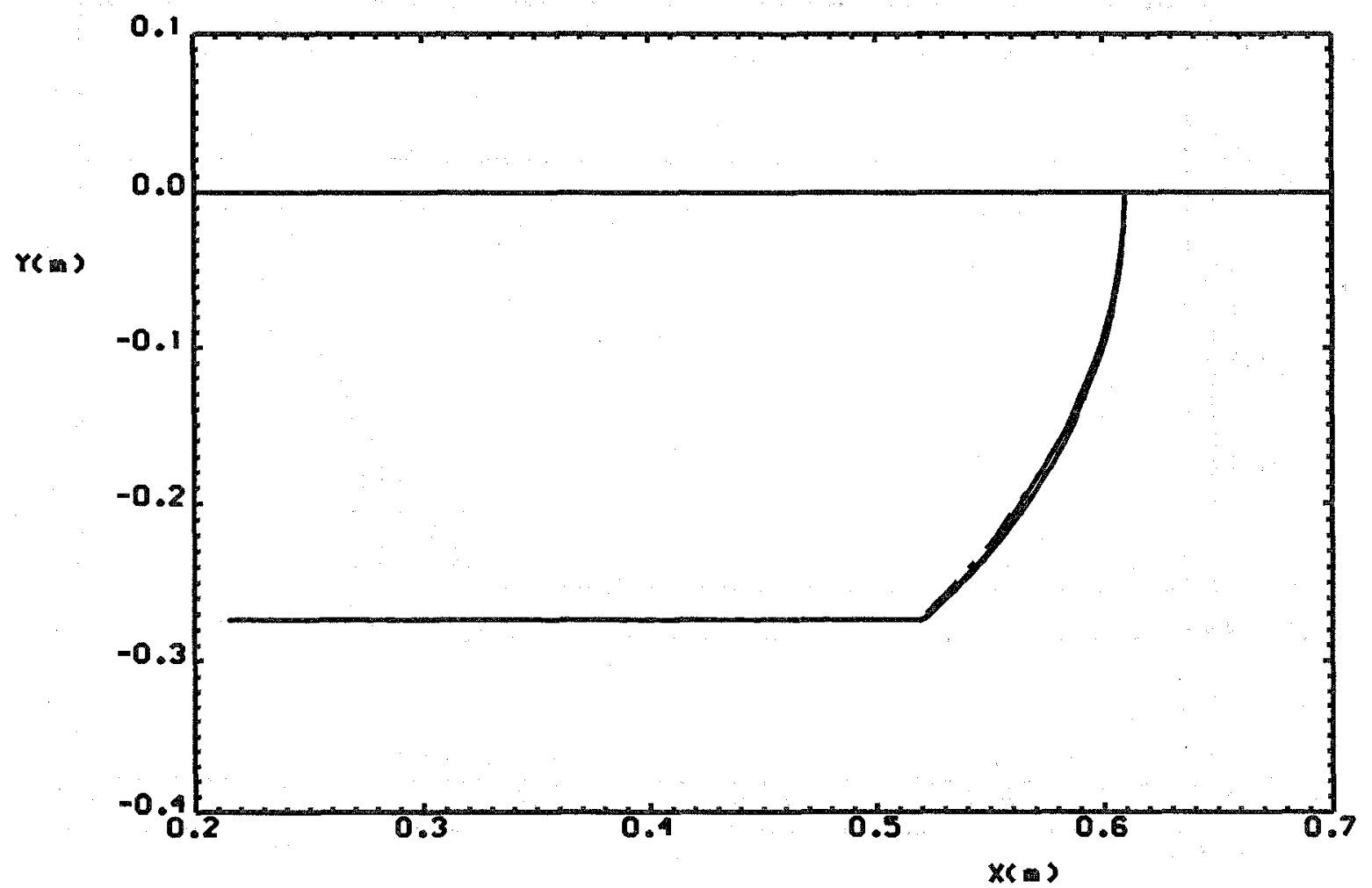

(b)

Figure 2: Work-space trajectory tracking performance (controller $\mathbf{C}_{2}$ )

(a) without payload (b) with payload 

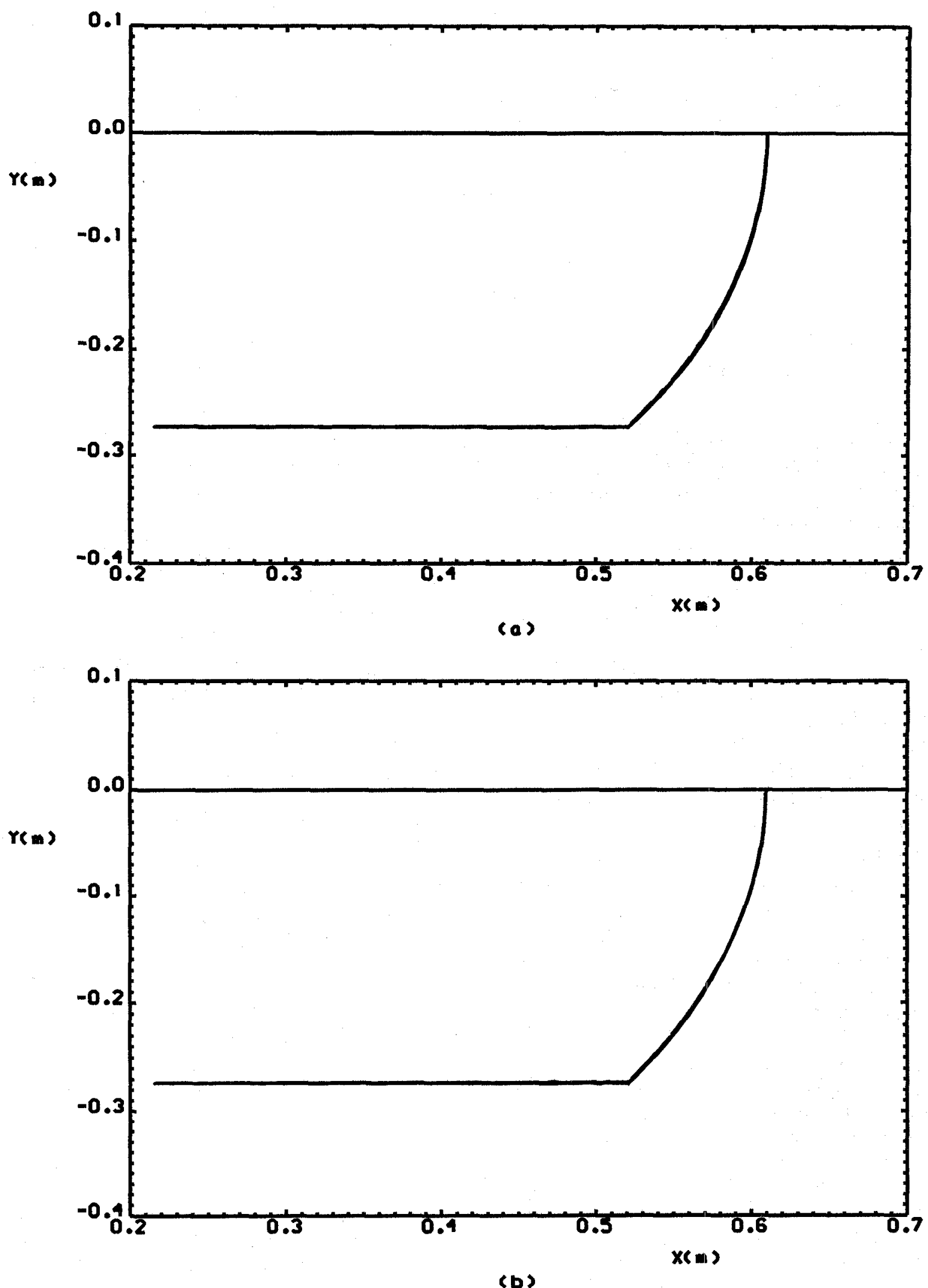

Figure 3: Work-space trajectory tracking performance (controller $\mathbf{C}_{3}$ )

(a) without payload (b) with payload 